\title{
Study of human liver disease with P-31 magnetic resonance spectroscopy
}

\author{
R Oberhaensli, B Rajagopalan, G J Galloway, D J Taylor, G K Radda
}

MRC Biochemical and Clinical Magnetic Resonance Unit, John Radcliffe Hospital, Oxford

R Oberhaensli

B Rajagopalan

G J Galloway

D J Taylor

G K Radda

Correspondence to: Dr B Rajagopalan, MRC Biochemical and Clinical Magnetic Resonance Unit, John Radcliffe Hospital, Headington, Oxford OX39DU.

Accepted for publication 3 July 1989

TABLE I Summary of clinical characteristics

\begin{tabular}{|c|c|c|c|c|c|}
\hline No & Age & Sex & $\begin{array}{l}\text { Bilirubin } \\
(\mu \mathrm{mol} / \mathrm{l}) \\
(3-17)\end{array}$ & $\begin{array}{l}A S T \\
(I U) \\
(10-35)\end{array}$ & $\begin{array}{l}\text { Alkaline } \\
\text { phosphatase } \\
(I U) \\
(100-300)\end{array}$ \\
\hline \multicolumn{6}{|l|}{ Primary biliary cirrhosis } \\
\hline 1 & 69 & $\mathbf{F}$ & 154 & 122 & 4740 \\
\hline 2 & 52 & $\mathrm{~F}$ & 357 & 159 & 836 \\
\hline 3 & 58 & $\mathrm{~F}$ & 23 & 109 & 426 \\
\hline \multicolumn{6}{|l|}{ Alcoholic hepatitis } \\
\hline 4 & 76 & $M$ & - & 116 & 219 \\
\hline 5 & 40 & $\mathrm{~F}$ & 12 & 58 & 113 \\
\hline 6 & 26 & M & 35 & 34 & 193 \\
\hline 7 & 45 & $\mathbf{F}$ & 98 & 109 & 542 \\
\hline \multicolumn{6}{|l|}{ Viral hepatitis } \\
\hline 8 & 46 & $\mathbf{M}$ & 133 & 2121 & 515 \\
\hline 9 & 23 & M & 237 & 1423 & 237 \\
\hline 10 & 28 & $M$ & 183 & 1819 & 622 \\
\hline 11 & 21 & $\ddot{M}$ & 285 & 333 & 749 \\
\hline 12 & 26 & $M$ & 350 & 78 & 696 \\
\hline \multicolumn{6}{|l|}{ Paracetamol overdose } \\
\hline entenge & $\begin{array}{l}16 \\
47\end{array}$ & $\underset{\mathrm{F}}{\mathrm{M}}$ & $\begin{array}{l}40 \\
20\end{array}$ & $\begin{array}{r}30 \\
9736\end{array}$ & $\begin{array}{l}316 \\
208\end{array}$ \\
\hline Haemochromatosis & & & & & \\
\hline 15 & 45 & $M$ & \multicolumn{3}{|c|}{ Ferritin $>3000 \mathrm{ng} / \mathrm{ml}$} \\
\hline $\begin{array}{l}16 \\
\text { Thalassaemias }\end{array}$ & 43 & $\mathbf{F}$ & \multicolumn{3}{|c|}{ Ferritin $>2000 \mathrm{ng} / \mathrm{ml}$} \\
\hline 17 & 16 & $M$ & \multirow{5}{*}{\multicolumn{3}{|c|}{$\begin{array}{l}\text { Ferritin } 1200 \mathrm{ng} / \mathrm{ml} \\
\text { Ferritin } 680 \mathrm{ng} / \mathrm{ml} \\
\text { Ferritin }>1000 \mathrm{ng} / \mathrm{ml} \\
\text { Ferritin } 740 \mathrm{ng} / \mathrm{ml} \\
\text { Liver iron } 1.33 \% \text { dry wt }(\text { normal }<0.016 \%)\end{array}$}} \\
\hline 18 & 28 & M & & & \\
\hline 19 & 16 & $\mathrm{~F}$ & & & \\
\hline 20 & 23 & M & & & \\
\hline & 18 & M & & & \\
\hline \multirow{4}{*}{$\begin{array}{l}\text { Tumours } \\
22 \\
23 \\
24\end{array}$} & & & & & \\
\hline & 65 & $M$ & 9 & 91 & 855 \\
\hline & 25 & $M$ & 15 & 13 & 281 \\
\hline & 54 & $\mathrm{~F}$ & 29 & 334 & 311 \\
\hline
\end{tabular}

Phosphorus-31 magnetic resonance spectros-

Abstract

Liver metabolism and energetics of 24 patients with liver disease were studied using phosphorus-31 magnetic resonance spectroscopy. Significant abnormalities were detected in the majority of these patients. A striking diversity in metabolic patterns was observed. Patients with acute viral hepatitis had low liver phosphodiesters and high phosphomonoesters, possibly phosphocholine and phosphoethanolamine. In alcoholic hepatitis phosphomonoesters were raised. Intracellular inorganic phosphate and inorganic phosphate/ATP ratios were decreased in primary biliary cirrhosis and in some patients with hepatitis. These spectroscopic results were evaluated in respect of the pattern of liver damage and cellular regeneration. Liver tumours had raised phosphomonoesters and also showed evidence for altered spin-lattice relaxation of the phosphorus nucleus in various metabolites. In iron overload the liver ATP resonances were broadened. The line broadening correlated with the degree of iron overload suggesting the potential use of P-31 magnetic resonance spectroscopy for measuring liver iron. which biochemical data can be non-invasively obtained from human tissues. Studies in animals have shown that in liver, ATP, inorganic phosphate, phosphomonoesters and phosphodiesters can be identified. In man, only studies on disorders of carbohydrate metabolism and of a few patients with primary and secondary liver tumours have been reported. ${ }^{1-4} \mathrm{P}-31$ spectroscopic data on experimental liver disorders in animals are also scarce. ${ }^{56}$

Both magnetic resonance imaging and spectroscopy utilise the interaction between a strong magnetic field and radiofrequency energy to detect nuclei such as ${ }^{1} \mathrm{H}$ or ${ }^{31} \mathrm{P}$. These nuclei have magnetic moments so that when placed in a magnetic field, they line up like little bar magnets. If a coil of wire is placed at right angles to the magnetic field over a sample and a brief radiofrequency pulse of the right frequency is passed through the coil, the nuclei are tipped over. As the nuclei realign themselves a small alternating current is induced in the coil. The frequency of this current is uniquely determined by the nucleus, its chemical environment and the strength of the magnetic field; and the amplitude by the number of nuclei present in the sample. By applying magnetic field gradients across the sample, the spatial distribution of the nuclei can also be determined. In magnetic resonance imaging, the signal from ${ }^{1} \mathrm{H}$ in all chemical compounds in tissues is used to generate the image. As water and fat contribute the major proportion of the protons, magnetic resonance images represent the density of tissues. In magnetic resonance spectroscopy, by using much stronger magnetic fields (typically $>1.5$ tesla) and magnets with much more homogenous fields, signals due to different phosphorus compounds can be resolved.

We have previously evaluated P-31 magnetic resonance spectroscopy for the metabolic assessment of normal human liver. ${ }^{78} \mathrm{We}$ report here results from common liver disorders investigated with this technique. Some of the results have been presented in preliminary form. ${ }^{9}$

\section{Methods}

PATIENTS

Twenty four patients with liver disease were selected for P-31 magnetic resonance spectros- 
copy of the liver. They were studied in the fasted state and their clinical profiles are summarised in Table I.

\section{ACUTE VIRAL HEPATITIS}

Five male patients were studied one to two weeks after onset of jaundice. One patient had mononucleosis, two hepatitis $B$, one hepatitis $A$, and in one the virus could not be identified. Patients had raised liver enzymes and were jaundiced. Plasma albumin and prothrombin time were normal.

\section{ALCOHOLIC HEPATITIS}

Four male chronic alcoholics were examined a few days after onset of symptoms. They were jaundiced and had raised liver enzymes. Liver biopsy showed fatty liver with predominantly perivenous cell damage.

\section{PRIMARY BILIARY CIRRHOSIS}

Three female patients with laboratory signs of intrahepatic cholestasis were investigated. Liver biopsy showed extensive periportal fibrosis and inflammatory reaction.

\section{LIVER TUMOURS}

One histologically proven hepatoblastoma, one secondary uterine carcinoma, and one secondary melanoma were studied. The tumours were several centimetres in diameter. Computerised tomography and ultrasound scans showed that they extended to the liver surface. A liver spectrum from one of the patients has been published. ${ }^{3}$

\section{IRON OVERLOAD}

Two patients had primary haemochromatosis and five had transfusional iron overload resulting from the treatment of thalassaemia. Liver biopsy sections (in the patients biopsied) stained for iron showed deposition of iron but no signs of liver cirrhosis. Patients were treated with an iron chelator (Desferal) and iron overload was monitored by measurements of plasma ferritin concentrations.

\section{MISCELLANEOUS DISORDERS}

Two patients were examined three to four days after an overdose of paracetamol. One patient was slightly jaundiced and had raised liver enzymes, the other was normal except for a small increase in AST.

TABLE II Liver metabolites. Absolute signal intensity in artibrary units

\begin{tabular}{|c|c|c|c|c|c|c|c|c|c|}
\hline & \multirow[b]{2}{*}{$n$} & \multicolumn{2}{|c|}{ Phosphomonesters } & \multicolumn{2}{|c|}{ Phosphodiesters } & \multicolumn{2}{|l|}{$P i$} & \multicolumn{2}{|l|}{$A T P$} \\
\hline & & mean & range & mean & range & mean & range & mean & range \\
\hline \multirow{5}{*}{$\begin{array}{l}\text { Controls } \\
\text { Viral hepatitis } \\
\text { Alcoholic hepatitis } \\
\text { Primary biliary } \\
\text { cirrhosis } \\
\text { Tumours }\end{array}$} & 16 & $1 \cdot 1$ & $0 \cdot 7-1 \cdot 5$ & $4 \cdot 5$ & $3 \cdot 0-6 \cdot 1$ & 1.8 & $1 \cdot 1-2 \cdot 5$ & $2 \cdot 5$ & $1 \cdot 7-3 \cdot 3$ \\
\hline & 5 & 1.9 & $1 \cdot 2-2 \cdot 7$ & $2 \cdot 6$ & $1 \cdot 3-3 \cdot 5$ & $1 \cdot 3$ & $0.7-1.8$ & $2 \cdot 0$ & $1.4-2.6$ \\
\hline & 4 & $1 \cdot 8$ & $1 \cdot 4-2 \cdot 4$ & $4 \cdot 8$ & $3 \cdot 7-6 \cdot 1$ & $1 \cdot 5$ & $1 \cdot 2-1 \cdot 7$ & $2 \cdot 4$ & $2 \cdot 0-3 \cdot 0$ \\
\hline & 3 & $1 \cdot 4$ & $1 \cdot 0-1 \cdot 5$ & $3 \cdot 5$ & $2 \cdot 7-5 \cdot 0$ & $0 \cdot 7$ & $0.6-0.8$ & 1.6 & $1 \cdot 3-1 \cdot 8$ \\
\hline & 3 & $3 \cdot 6$ & $3 \cdot 1-4 \cdot 1$ & $3 \cdot 8$ & $3 \cdot 2-4 \cdot 3$ & 1.9 & $1 \cdot 7-2 \cdot 1$ & 2.6 & $2 \cdot 2-3 \cdot 0$ \\
\hline
\end{tabular}

\section{CONTROL SUBJECTS}

Four women and 12 men aged $25-43$ years acted as controls. They were normal on routine clinical and laboratory assessment. The study protocol was approved by the local Ethics Committee. Informed consent was obtained from all subjects.

\section{P-3 I MAGNETIC RESONANCE SPECTROSCOPY}

The magnetic resonance spectroscopy investigations were done as previously described ${ }^{8}$ Spectra were obtained at 1.9 tesla using an $8 \mathrm{~cm}$ diameter surface coil. After determining the position of the liver by percussion and, in some cases, ultrasound, the patient was positioned on the right side with the centre of the liver dullness (usually over the mid to anterior axillary line) over the centre of the coil. In patients with liver tumours the surface coil was positioned over the tumour; the location of the tumours was determined from computed tomography and ultrasound scans. Signal from the liver was localised using magnetic field profiling. ${ }^{8}$ In this technique magnetic field homogeneity was first optimised using the proton signal from water over a sphere $10 \mathrm{~cm}$ in diameter. The homogenous part of the magnetic field was then reduced to a sphere $5 \mathrm{~cm}$ in diameter situated in the centre of the original volume. In most subjects this procedure excluded signal from the chest wall. Signal from a $30 \mathrm{cc}$ volume of liver was obtained. A $180^{\circ}$ pulse at coil centre and an interpulse delay of either $1 \mathrm{~s}$ ( 256 or 512 transients) or $0 \cdot 1$ s ( 2560 transients) were used. Total study time including positioning of the patient took about 30 minutes. The transients (free induction decays) were processed by deconvolution to remove the large underlying hump inherent to the field profiling experiment, then multiplied by an exponential line broadening function of $15 \mathrm{~Hz}$ before fourier transformation. The phase of the resulting spectra were manually corrected. Peak areas were calculated by triangulation of spectra plotted with a standard set of parameters.

In two-thirds of the examinations, the signals obtained were exclusively from liver as indicated by the absence of any phosphocreatine signal (phosphocreatine is a major constituent of the overlying skeletal muscle but is absent in liver). Even when a small phosphocreatine peak was present in the spectra, indicating some contamination from muscle, its area was always less than $50 \%$ of the $\gamma$-ATP peak. From known metabolite ratios in skeletal muscle ${ }^{10}$ it can be calculated that muscle metabolites contributed less than $10 \%$ to the peak areas of ATP, inorganic phosphate, phosphomonoesters and phosphodiesters. Though we refer to ATP peaks, studies on extracts of animal livers have shown that up to $10 \%$ of the signal may be derived from other nucleotide triphosphates such as uridine triphosphate."

The problems of measuring absolute concentrations of metabolites from magnetic resonance spectroscopy spectra obtained using surface coils are well known. ': In the present study, interpretation of the data was based on ratios of peak areas. We found that when the absolute areas of 
peaks in the control studies were measured, however, the coefficient of variation for ATP was about $15 \%$. This small variation was seen presumably because using the same protocol, processing and plotting parameters, the signal obtained in most subjects, was derived from a similar volume of liver. We therefore summarised the absolute signal intensities in Table II as an adjunct to the ratios in Figure 2. The units for the absolute signal intensities were derived assuming that the mean of the intensities of the $\beta$-ATP peak in 16 control subjects represented $2.5 \mu \mathrm{mol} / \mathrm{g}$ wet wt. (This is the concentration of ATP in normal human liver as determined by direct biochemical assay ${ }^{13}$.

Neither metabolite ratios nor absolute signal intensities were corrected for saturation effects because of incomplete $T_{1}$ relaxation. In a magnetic resonance spectroscopy experiment, the recovery of the phosphorus nuclei to equilibrium after a radiofrequency pulse is an exponential process determined by $T_{1}$. Interpulse delays shorter than approximately five times $T_{1}$ will lead to incomplete recovery or relaxation and hence a reduction in signal. We determined if there were any changes in $T_{1}$ by comparing peak areas from spectra obtained using repetition rates of $0.1 \mathrm{~s}$ and $1 \mathrm{~s}$. In healthy subjects under the pulse conditions used $\left(180^{\circ}\right.$ pulse at coil centre and interpulse delay of $1 \mathrm{~s}$ ) ATP was fully relaxed whereas phosphomonoesters, inorganic phosphate and phosphodiesters were about $70 \%$, $80 \%$ and $50 \%$, respectively, of the full unsaturated signal. ${ }^{8}$ The effect of $T_{1}$ relaxation on peak intensities was estimated by calculating the ratio of signal intensity at $0.1 \mathrm{~s}$ interpulse delay to signal intensity at $1.0 \mathrm{~s}$ interpulse delay. This ratio we define as the ' $T_{1}$ relaxation factor'. Chemical shift was measured relative to water and expressed as parts per million (ppm) relative to phosphocreatine..$^{78}$ The $\mathrm{pH}$ was derived from the shift of inorganic phosphate. ${ }^{10}$ The control range is defined as mean \pm 2 standard deviations.

\section{Results}

Figure 1 shows P-31 liver spectra from a control subject and three patients with liver diseases. It can be seen that there are differences in relative peak intensities in the spectra. The ratios of the various liver metabolites are given in Figure 2. All patients had raised liver phosphomonoesters/ ATP ratio except for the one patient with paracetamol overdose and minimal laboratory evidence of liver damage. The liver inorganic phosphate/ATP ratio was decreased in patients with primary biliary cirrhosis and in some patients with viral or alcoholic hepatitis. The liver phosphodiesters/ATP ratio was reduced in all patients with viral hepatitis. It was variable in alcoholic hepatitis and tumours. Intracellular liver $\mathrm{pH}$ was normal (range: $7 \cdot 1-7 \cdot 4$ ) in all patients except for one with primary biliary cirrhosis; her liver $\mathrm{pH}$ was greater than $7 \cdot 5$.

The absolute signal intensities of the liver metabolites are summarised in Table II. Patients with acute viral hepatitis had high normal (one) to increased (four) liver phosphomonoesters and low normal (two) to decreased (three) phosphodiesters. Patients with alcoholic hepatitis

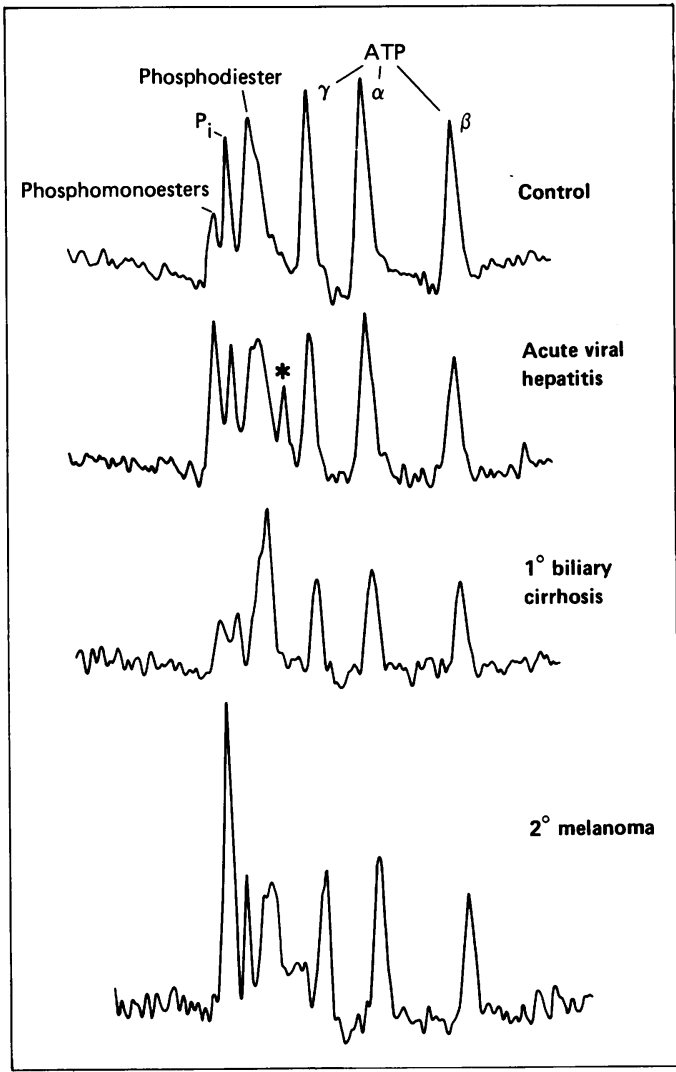

Figure 1: P-31 liver spectra of patients with liver disease. Phosphomonoester: phosphocholine and

phosphoethanolamine, phosphorylated sugars:

Phosphodiesters, probably glycerophosphorylcholine, glycerophosphorylethanolamine, phosphatidylcholine and phosphatidylethanolamine, Pi: inorganic phosphate (intracellular); ATP: resonances of the $\alpha, \beta$ and $\gamma$ phosphate of purine and pyrimidine nucleotides, mainly ATP. The $\alpha-A T P$ peak also contains contributions from $N A D(H)$ and UDP-glucose. Phosphocreatine due to small contamination of the spectrum with signals from skeletal muscle (diaphragm or intercostals) is denoted by the asterisk.

also had high normal (one) to increased (four) liver phosphomonoesters and low normal (two) to decreased (three) phosphomonoesters. Patients with alcoholic hepatitis also had high normal (one) or increased (three) liver phosphomonoesters but phosphodiesters were normal. All patients with primary biliary cirrhosis showed a marked reduction in liver inorganic phosphate. In two the ATP was also decreased. Phosphomonoesters were high in liver tumours.

There were differences in the chemical shift of the phosphomonoesters peaks in the spectra from some of the patients when compared with controls. The chemical shift of the phosphomonoesters peak depends on the relative contribution of its constituents, phosphocholine, phosphoethanolamine and sugar phosphates. At a field of $1.9 \mathrm{~T}$, however, individual components cannot be resolved.

The $T_{1}$ relaxation factors of the patients with hepatoblastoma and $2^{\circ}$ uterine carcinoma were different from the values in the controls $\left(T_{1}\right.$ relaxation factors in controls: phosphomonoesters $=0.35$ (range $0 \cdot 20-0.54)$, inorganic phosphate $=0.46$ (range $0.31-0.75)$, phosphodiesters $=0.32$ (range $0.18-0.48), \beta A T P=0.51$ (range $0 \cdot 40-0 \cdot 65$ ). Hepatoblastoma: phosphomonoesters $=0 \cdot 18$, phosphate $=0 \cdot 18$, phosphodiesters $=0 \cdot 36, \beta \mathrm{ATP}=0 \cdot 24: 2^{\circ}$ uterine carcinoma: phosphomonoesters $=0.41$, inorganic 

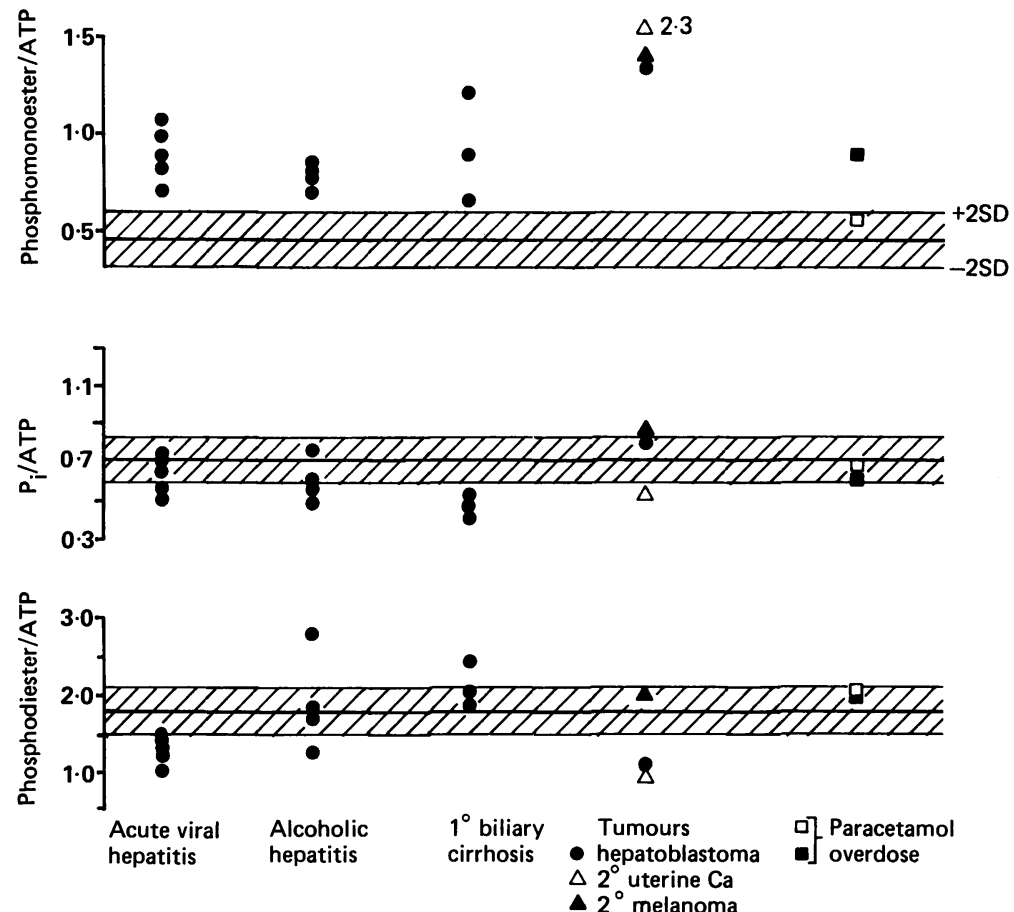

Figure 2: Ratios of liver metabolites. Control range: mean \pm 2 standard deviations $(n=16)$.

phosphate $=0.69, \quad$ phosphodiesters $=0.55$, $\beta \mathrm{ATP}=0.47$.

P-31 liver spectra of the patients with iron overload are shown in Figure 4. The peaks were broadened in moderate iron overload (plasma ferritin $675 \mathrm{mg} / \mathrm{ml}$ ) and were broadened out completely in severe iron overload (plasma ferritin $3750 \mathrm{mg} / \mathrm{ml}$ ). Well resolved P-31 spectra from overlying muscle were obtained in these patients indicating that the line broadening was the result of changes in relaxation times and not the result of technical problems. The water peak from the liver proton spectrum was also markedly broadened in these patients (data not shown).

\section{Discussion}

This study shows that abnormalities in liver metabolism can be detected by P-31 magnetic resonance spectroscopy. The signal in the phosphomonoesters region was raised in 22 of the 25 patients. The abnormal phosphomonoesters peaks were found in all patients with viral

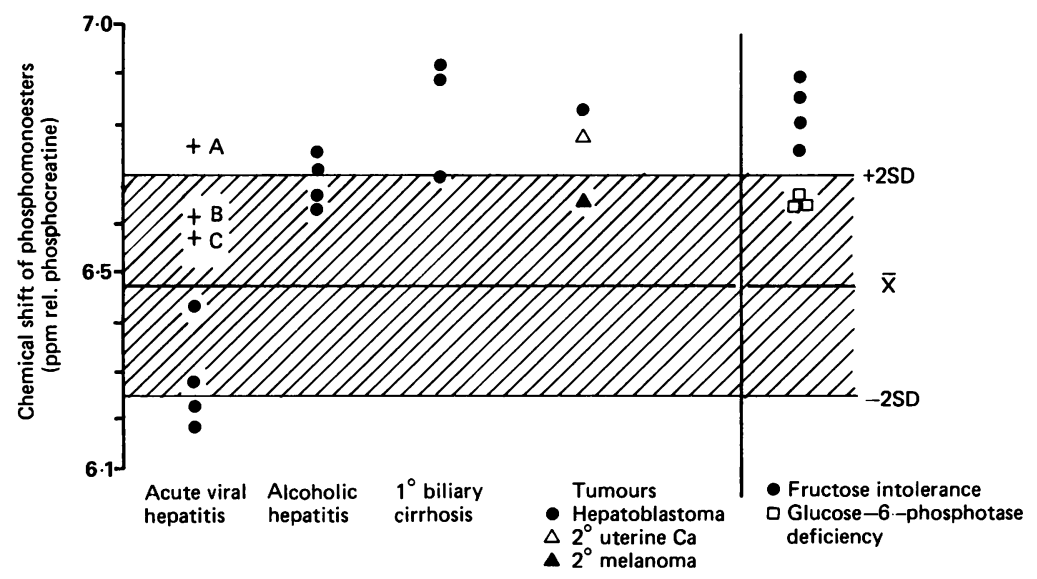

Figure 3: Chemical shift of phosphomonoesters resonance. (Repeat examinations in patient with infectious mononucleosis: $B$ three days and $C$ five days after examination A.) Control range: mean \pm 2 standard deviations $(n=16)$.

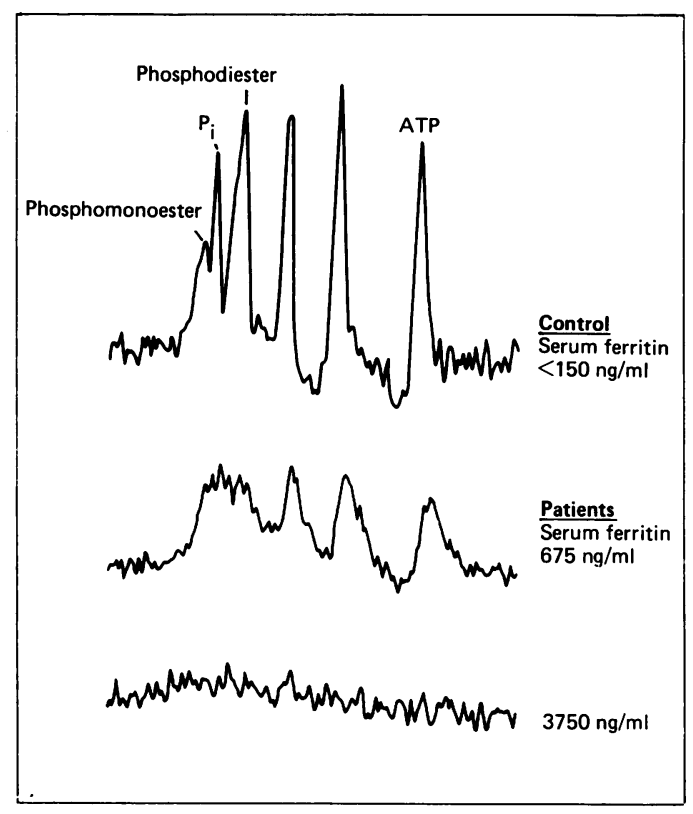

Figure 4: $P$-31 liver spectra of patients with iron overload. Peak assignment as in Figure 1 .

hepatitis, alcoholic hepatitis, primary biliary cirrhosis and one of the patients with paracetamol overdose. The signal in the phosphodiesters region was reduced in patients with acute viral hepatitis. The inorganic phosphate/ ATP was low in the patients with primary biliary cirrhosis. None of the spectra showed raised inorganic phosphate/ATP and only one showed an abnormal intracellular $\mathrm{pH}$. The peaks in the spectra from patients with iron overload were broadened so that it was difficult or impossible to measure peak areas.

What is the nature of the compounds which make up the phosphomonoesters peak? Liver extracts examined at higher fields show that sugar phosphates such as glucose or fructose phosphate, and compounds such as phosphoethanolamine and phosphocholine can generate signals in the phosphomonoesters region of the spectrum." Phosphoethanolamine and phosphocholine are intermediates in phospholipid biosynthesis and have been shown to be high during rapid cell membrane biosynthesis. ${ }^{14}$

In the patients with viral hepatitis the high phosphomonoesters which returned to normal as liver function became normal were probably associated with liver regeneration one to two weeks after the onset of the jaundice when the investigations were performed. Studies have shown that increased phosphomonoesters are present in regenerating rat liver and in some human liver tumours, and analysis of extracts of these tissues has shown that this increase is the result of phosphoethanolamine. ${ }^{+15}$ Increases in phosphomonoesters were also seen in patients with alcoholic hepatitis. Raised sugar phosphates after acute ethanol administration have been previously documented. ${ }^{16}$ Patients with inborn errors of carbohydrate metabolism show increases in sugar phosphates in extracts of their liver biopsies thus confirming that in some conditions sugar phosphates may contribute significantly to the phosphomonoesters peak. This has been strikingly shown by P-31 magnetic 
resonance spectroscopy in both homozygotes and heterozygotes with fructose intolerance who show a large fructose phosphate peak after a fructose load.' Further studies are necessary to determine the relative contributions of phospholipid precursors and sugar phosphates to the increased phosphomonoesters signal in spectra of patients.

The signal in the phosphodiesters region in liver spectra has not yet been assigned conclusively. Studies on perfused liver and on brain extracts suggest that it is probably a composite of signals from several classes of compounds. ${ }^{11}$. In the perfused rat liver, resonances in this region were assigned to products of phospholipid breakdown such as glycerophosphorylcholine and glycerophosphorylethanolamine, " while in brain phosphatidylcholine and phosphatidylethanolamine have also been detected. ${ }^{17}$ Thus the decrease in phosphodiesters which we observe in acute hepatitis could be caused by low concentrations of these compounds indicating reduced membrane breakdown.

The intracellular inorganic phosphate and inorganic phosphate/ATP reflect the bioenergetic state of the cell. It was surprising that none of the patients showed any evidence of increased inorganic phosphate/ATP or a high inorganic phosphate in view of the liver cell damage that they were known to have suffered. Ischaemic or hypoxic cells in brain, heart, kidney, ${ }^{5}$ and liver show increased inorganic phosphate levels and inorganic phosphate/ATP ratios. The findings in our patients imply that dying cells, if present, contributed little to P-31 spectra we obtained. Some patients had a low inorganic phosphate to ATP ratio even though plasma inorganic phosphate was normal. It is not known whether the low inorganic phosphate is due to loss of liver inorganic phosphate from the cells or to the shift of inorganic phosphate into magnetic resonance invisible pools which comprise about $50 \%$ of normal liver inorganic phosphate. ${ }^{8}$ At most the low inorganic phosphate/ATP is likely to be a non-specific indicator of liver cell damage.

In the patients with iron overload the resonances of P-31 liver spectra were broadened apparently depending on the degree of iron overload. The line broadening could result in part from susceptibility effects of $\mathrm{Fe}^{3+}$ which is stored as ferritin and haemosiderin in liver lysosomes. ${ }^{18} 19$ Similar broadening of proton lines in liver have been observed using proton imaging in patients with haematomas or haemochromatosis. ${ }^{2021}$

In conclusion, we have shown several biochemical abnormalities in diseased liver by P-31 magnetic resonance spectroscopy. Some of the results may allow new insight into membrane breakdown and biosynthesis in liver disease.
Although the information gained is not as yet directly relevant to the day to day management of patients, it may contribute to the better understanding of liver disease and its therapy.

This study was supported by the Medical Research Council and the Department of Health and Social Security of Great Britain RDO received a personal grant from the Swiss National Science Foundation. We thank our colleagues from the John Radcliffe Hospital and Churchill Hospital, Oxford, for allowing us to study their patients. Two tumour patients were studied in collaboration their patients. Two tumour patients were stud
with $\mathrm{Mr}$ Peter Bore and Miss Linda Hands.

1 Oberhaensli RD, Rajagopalan B, Taylor DJ, et al. Study of hereditary fructose intolerance using ${ }^{31} \mathrm{P}$ magnetic resonance spectroscopy. Lancet 1987; ii: 931-4.

2 Oberhaensli RD, Rajagopalan B, Taylor DJ, Radda GK Collins JE, Leonard JV. Study of liver metabolism in glucose-6-phosphatase deficiency (glycogen storage disease type $1 \mathrm{~A})$ by P-31 magnetic resonance spectroscopy. Pediat Res 1987; 23: 375-80.

3 Oberhaensli RD, Hilton-Jones D, Bore PJ, Hands LJ, Rampling RP, Radda GK. Biochemical investigation of human tumours in vivo with phosphorus-31 magnetic human tumours in vivo with phosphorus-

4 Maris JM, Evans AE, McLaughlin AC, et al. "P nuclear magnetic resonance spectroscopic investigation of huma neuroblastoma in situ. N Englf Med 1985; 312: 1500-5.

5 Radda GK, Taylor DJ. Applications of nuclear magnetic resonance spectroscopy in pathology. In: Richter $G$ W Epstein MA, eds. International review of experimental pathology. New York \& London: Academic Press, 1985; $1-58$.

6 Bates TF, Williams SR, Gadian DG, et al.A "P nuclear magnetic resonance study in vivo of metabolic abnormalities

7 Oberhaensli RD, Galloway GJ, Taylor DJ, Bore PJ, Radd GK. Assessment of human liver metabolism by phosphorus31 magnetic resonance spectroscopy. Br $\mathcal{F}$ Radiol 1986; 59: 695-9.

8 Oberhaensli RD, Galloway GJ, Hilton-Jones D, et al. The study of human organs by phosphorus-31 topical magnetic resonance spectroscopy. Br $\mathcal{F}$ Radiol 1987; 60: 367-73.

9 Oberhaensli RD, Galloway GJ, Taylor DJ, Bore PJ, Rajagopalan B, Radda GK. First year of experience with P-3 magnetic resonance studies of human liver. Magn Reson Imaging 1986; 4: 413-6.

10 Taylor DJ, Bore PJ, Styles P, Gadian DG, Radda GK. Bioenergetics of intact human muscle: A "P nuclear magnetic resonance study. Mol Biol Med 1983; 1: 77-94.

11 Cohen SM. Simultaneous ${ }^{13} \mathrm{C}$ and ${ }^{31} \mathrm{P}$ NMR studies of perfused rat liver. Effects of insulin and glucagon and a ${ }^{13} \mathrm{C}$ NMR rat liver. Effects of insulin and glucagon and a 'C

12 Evelhoch JE, Crowley MG, Ackerman JJH. Signal to noise optimisation and research localisation with circular surface coils. F Magn Reson 1984; 56: 110-24.

13 Hultman E, Nilsson LH, Sahlin K. Adenine nucleotide content of human liver. Normal values and fructose induced depletion. Scand F Clin Lab Invest 1975; 35: 245-51.

14 Pelech SL, Vance DE. Regulation of phosphatidylcholin biosynthesis. Biochim Biophys Acta 1984: 779: 217-51

15 Murphy E, Rajagopalan B, Brindle K, Radda GK. Phospholipid metabolism in regenerating rat liver: $A{ }^{31} \mathrm{P}$ magnetic resonance spectroscopy study. Clin Sci 1988; 74 (suppl 18):

16 Desmoulin F, Canionia P, Crotte C, Gerolami A, Cazzne PJ. Hepatic metabolism during acute ethanol administration: A phosphorus-31 nuclear magnetic resonance study on the perfused rat liver under normoxic and hypoxic conditions. perfused rat liver under norm
Hepatology 1987; 7: 315-23.

17 Cerdan S, Subramanian VH, Cone KHJ, Egan J, Chance B, Williamson JR. ${ }^{31} \mathrm{P}$ NMR detection of mobile dog brain phospholipids. Magn Red Med 1986; 3: 432-9.

18 Munro HN, Linder MC. Ferritin: Biosynthesis, and role in iron metabolism. Physiol Rev 1978; 58: 317-96.

19 Wixon RL, Prutkin L, Munro HN. Hemosiderin: Nature, formation and significance. Int Rev Exp Path 1980; 22: 193-227.

20 Stark DD, Moseley ME, Bacon BR, et al. Magnetic resonance imaging and spectroscopy of hepatic iron overload. Radiology 1985; 154: 137-42.

21 Felder R, Stark JD, Bacon BR, Lauffer R, Brady TJ. MR imaging of systemic iron overload. Radiology 1985; 157: 297. 